\title{
Erratum: Tests of General Relativity with GW150914 [Phys. Rev. Lett. 116, 221101 (2016)]
}

\author{
B. P. Abbott et al.
}

\author{
(LIGO Scientific and Virgo Collaboration) $)^{* \dagger, *}$
}

(Received 20 July 2018; published 18 September 2018)

DOI: $10.1103 /$ PhysRevLett.121.129902

This Erratum reports an error found in the implementation of the code of the LIGO Scientific and Virgo Collaborations (LVC) as used in gravitational-wave-based estimations of possible deviations from the post-Newtonian (PN) terms expected in general relativity (GR). The error concerned the $0.5 \mathrm{PN}$ term and affected the results previously published for GW150914 [1] in Ref. [2], for GW151226 [3] in Ref. [4], and for GW170104 in Ref. [5]. We corrected the bug and present the reproduced results in this Erratum, as well as in the related Errata [6,7]. The main conclusion, that the results are consistent with general relativity, remains.

In Ref. [2], the test for the parameterized post-Newtonian [8] deviations from the expected GR values relied on creating non-GR waveforms [9-13] and using them as potential matches for the observed waveforms [14-17]. In these waveforms, implemented in the frequency domain, freedom was introduced by allowing the phase coefficients describing different powers of the post-Newtonian parameter (equivalently, powers of the frequency) to assume a range of values, not only the particular values prescribed by GR.

However, a coding bug was introduced, identically zeroing the deviations at $0.5 \mathrm{PN}$ in the inspiral regime (as in GR). The $0.5 \mathrm{PN}$ deviations were hence absent in the phasing formula, though not in the junction conditions that relate the inspiral regime to the intermediate regime. Any constraints obtained in $[2,4,5]$ only resulted from the latter.

This error affected the results of the non-GR parameter estimation (PE) [14] pipeline tests performed for finding bounds on possible PN deviations from GR. In particular, they affect the bounds on the single deviations in the $0.5 \mathrm{PN}$ term and on the tests with multiple deviations together. These erroneous results appeared in Figs. 6 and 7 and Table I of [2], in Figs. 7 and 8 of [4], and in Fig. 9 of the Supplemental Material of [5]. The corrected versions of all of these have been produced. The corrections for Figs. 6 and 7 and Table I of [2] appear below, while the others are available in [6,7]. All these results are consistent with GR.

The error, introduced by erroneous caching during the optimization of the waveform generation for efficient PE, has been corrected in commit [18] of the LALSuite [19] code. No subsequent LVC papers have been affected.

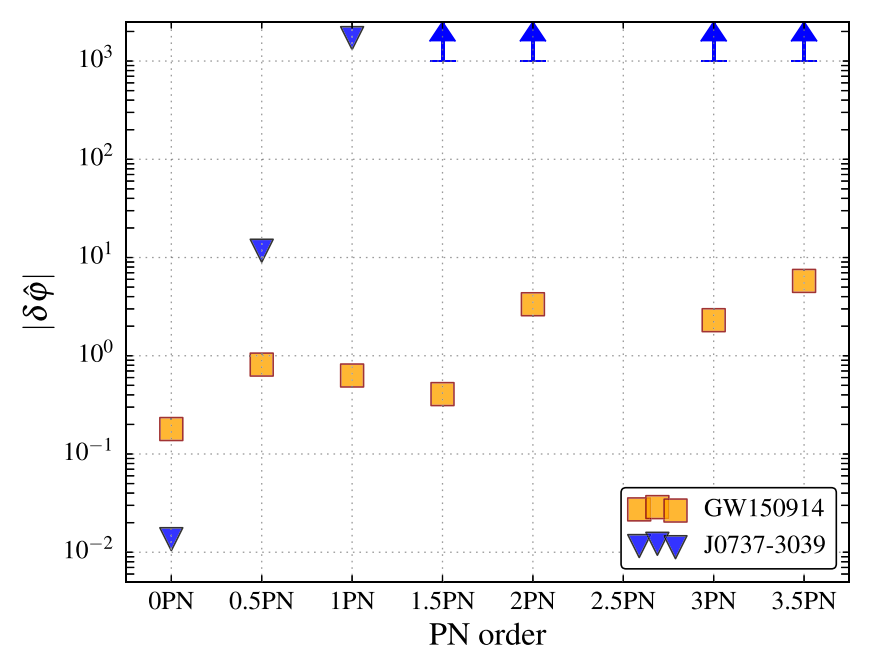

FIG. 1. This is the corrected Fig. 6 of [2], comparing the 90\% upper bounds on PN parameter deviations from GR obtained from the double pulsar PSR J0737-3039 [22,23] to those obtained from the first gravitational wave GW150914. 


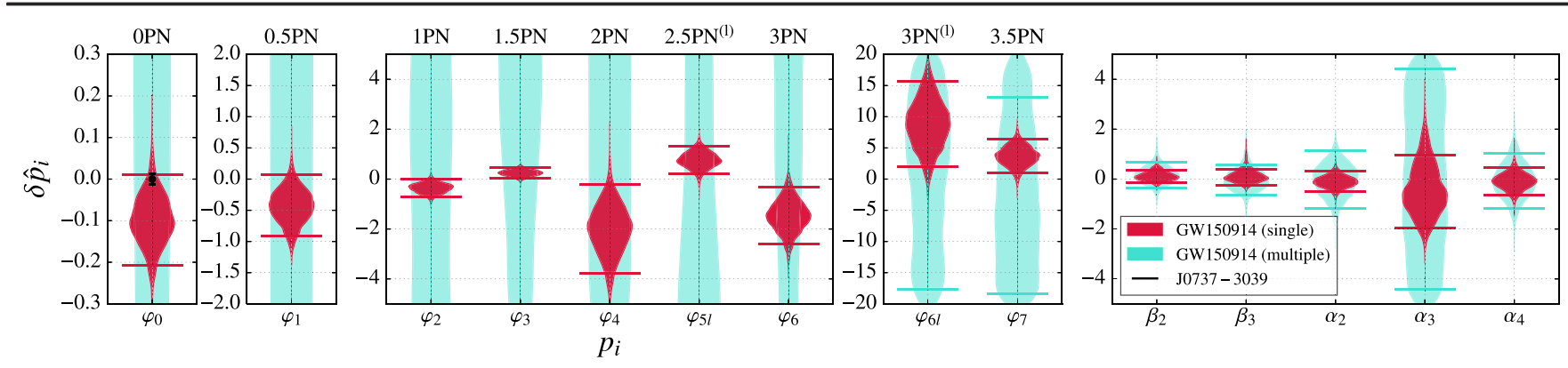

FIG. 2. This is the corrected Fig. 7 of [2], summarizing in a violin plot the posterior probability density distributions for all of the parameters in the GIMR model. The error bars indicate the $90 \%$ credible regions. The leftmost posteriors, labeled from 0 to 3.5 PN, are for the early-inspiral PN regime; the $\beta_{i}$ and $\alpha$ parameters correspond to the intermediate and merger-ringdown regimes. In red, we show posterior probability distributions for the single-parameter analysis, while in cyan we show the posterior distribution for the multipleparameter analysis. The corrected posterior for the $0.5 \mathrm{PN}$ single-parameter analysis peaks below zero, while previously reported to peak above; the posterior is still consistent with GR. The corrected multiple-parameter analysis no longer shows the erroneous special behavior for the $0.5 \mathrm{PN}$ term. The black error bar at $0 \mathrm{PN}$ shows the bound inferred from the double pulsar.

TABLE I. This is the corrected version of the second row of Table I of [2], namely, the results for $\delta \hat{\varphi}_{1}$ of the early-inspiral regime. Not shown in the table is the single result for the $\log _{10} B_{\text {model }}^{\mathrm{GR}}$ of the multiple-parameter early-inspiral regime comparison, for which the corrected value is $2.1 \pm 0.6$.

\begin{tabular}{|c|c|c|c|c|c|c|c|}
\hline \multirow[b]{2}{*}{ Waveform regime } & \multirow[b]{2}{*}{ Parameter } & \multirow[b]{2}{*}{$f$ dependence } & \multicolumn{2}{|c|}{ Median } & \multicolumn{2}{|c|}{ GR quantile } & \multirow{2}{*}{$\frac{\log _{10} B_{\text {model }}^{\mathrm{GR}}}{\text { Single }}$} \\
\hline & & & Single & Multiple & Single & Multiple & \\
\hline Early-inspiral regime & $\delta \hat{\varphi}_{1}$ & $f^{-4 / 3}$ & $-0.4_{-0.9}^{0.0}$ & $-0.6_{-18.0}^{+17.7}$ & 0.94 & 0.52 & $1.3 \pm 0.3$ \\
\hline
\end{tabular}

Note that, while this error also affected the analysis of GW170608 [20], the reported results require no changes: with the corrected analysis, the GR-predicted PN coefficient values continue to be consistent with the data. No change is required regarding the preliminary reported results for GW170814 [21] either.

*Full author list given at the end of the original Letter.

†1sc-spokesperson@ligo.org

*Virgo-spokesperson@ego-gw.it

[1] B. P. Abbott et al. (LIGO Scientific and Virgo Collaborations), Observation of Gravitational Waves from a Binary Black Hole Merger, Phys. Rev. Lett. 116, 061102 (2016).

[2] B. P. Abbott et al. (LIGO Scientific and Virgo Collaborations), Tests of General Relativity with GW150914, Phys. Rev. Lett. 116, 221101 (2016).

[3] B. P. Abbott et al. (LIGO Scientific and Virgo Collaborations), GW151226: Observation of Gravitational Waves from a 22-SolarMass Binary Black Hole Coalescence, Phys. Rev. Lett. 116, 241103 (2016).

[4] B. P. Abbott et al. (LIGO Scientific and Virgo Collaborations), Binary Black Hole Mergers in the First Advanced LIGO Observing Run, Phys. Rev. X 6, 041015 (2016).

[5] B. P. Abbott et al. (LIGO Scientific and Virgo Collaborations), GW170104: Observation of a 50-Solar-Mass Binary Black Hole Coalescence at Redshift 0.2, Phys. Rev. Lett. 118, 221101 (2017).

[6] LIGO Scientific and Virgo Collaborations, published concurrently, Erratum: Binary Black Hole Mergers in the First Advanced LIGO Observing Run, Phys. Rev. X 8, 039903 (2018).

[7] LIGO Scientific and Virgo Collaborations, following Erratum, Erratum: GW170104: Observation of a 50-Solar-Mass Binary Black Hole Coalescence at Redshift 0.2, Phys. Rev. Lett. 121, 129901 (2018).

[8] L. Blanchet, Gravitational radiation from post-Newtonian sources and inspiralling compact binaries, Living Rev. Relativity 17, 2 (2014).

[9] C. K. Mishra, K. G. Arun, B. R. Iyer, and B. S. Sathyaprakash, Parametrized tests of post-Newtonian theory using Advanced LIGO and Einstein Telescope, Phys. Rev. D 82, 064010 (2010).

[10] M. Agathos, W. Del Pozzo, T. G. F. Li, C. Van Den Broeck, J. Veitch, and S. Vitale, TIGER: A data analysis pipeline for testing the strong-field dynamics of general relativity with gravitational wave signals from coalescing compact binaries, Phys. Rev. D 89, 082001 (2014). 
[11] L. Sampson, N. Cornish, and N. Yunes, Gravitational wave tests of strong field general relativity with binary inspirals: Realistic injections and optimal model selection, Phys. Rev. D 87, 102001 (2013).

[12] T. G. F. Li W. Del Pozzo, S. Vitale, C. Van Den Broeck, M. Agathos, J. Veitch, K. Grover, T. Sidery, R. Sturani, and A. Vecchio, Towards a generic test of the strong field dynamics of general relativity using compact binary coalescence, Phys. Rev. D 85, 082003 (2012).

[13] T. G. F. Li, W. Del Pozzo, S. Vitale, C. Van Den Broeck, M. Agathos, J. Veitch, K. Grover, T. Sidery, R. Sturani, and A. Vecchio, Towards a generic test of the strong field dynamics of general relativity using compact binary coalescence: Further investigations, J. Phys. Conf. Ser. 363, 012028 (2012).

[14] B. P. Abbott et al. (LIGO Scientific and Virgo Collaborations), Properties of the Binary Black Hole Merger GW150914, Phys. Rev. Lett. 116, 241102 (2016).

[15] A. B. Nielsen, Compact binary coalescence parameter estimations for 2.5 post-Newtonian aligned spinning waveforms, Classical Quantum Gravity 30, 075023 (2013).

[16] J. Veitch et al., Parameter estimation for compact binaries with ground-based gravitational-wave observations using the LALInference software library, Phys. Rev. D 91, 042003 (2015).

[17] S. Vitale, Multiband Gravitational-Wave Astronomy: Parameter Estimation and Tests of General Relativity with Space- and Ground-Based Detectors, Phys. Rev. Lett. 117, 051102 (2016).

[18] https://git.ligo.org/lscsoft/lalsuite/commit/980b3f788a445d135390eba8861b03f70133cd47.

[19] LSC Algorithm Library Suite, https://wiki.ligo.org/DASWG/LALSuite, https://git.ligo.org/lscsoft/lalsuite.

[20] B. P. Abbott et al. (LIGO Scientific and Virgo Collaborations), GW170608: Observation of a 19-solar-mass binary black hole coalescence, Astrophys. J. 851, L35 (2017).

[21] B. P. Abbott et al. (LIGO Scientific and Virgo Collaborations), GW170814: A Three-Detector Observation of Gravitational Waves from a Binary Black Hole Coalescence, Phys. Rev. Lett. 119, 141101 (2017).

[22] N. Wex, Testing Relativistic Gravity with Radio Pulsars, arXiv:1402.5594.

[23] A. Maselli, S. Marassi, V. Ferrari, K. Kokkotas, and R. Schneider, Constraining Modified Theories of Gravity with GravitationalWave Stochastic Backgrounds, Phys. Rev. Lett. 117, 091102 (2016). 